\title{
Sensors 4.0 - smart sensors and measurement technology enable Industry 4.0
}

\author{
Andreas Schütze ${ }^{1,2}$, Nikolai Helwig ${ }^{2}$, and Tizian Schneider ${ }^{2}$ \\ ${ }^{1}$ Lab for Measurement Technology, Department Systems Engineering, Saarland University, \\ 66123 Saarbruecken, Germany \\ ${ }^{2}$ Centre for Mechatronics and Automation Technology (ZeMA gGmbH), 66121 Saarbruecken, Germany \\ Correspondence: Andreas Schütze (schuetze@1mt.uni-saarland.de)
}

Received: 13 November 2017 - Accepted: 25 February 2018 - Published: 9 May 2018

\begin{abstract}
Industrie 4.0" or the Industrial Internet of Things (IIoT) are two terms for the current (r)evolution seen in industrial automation and control. Everything is getting smarter and data generated at all levels of the production process are used to improve product quality, flexibility, and productivity. This would not be possible without smart sensors, which generate the data and allow further functionality from self-monitoring and selfconfiguration to condition monitoring of complex processes. In analogy to Industry 4.0, the development of sensors has undergone distinctive stages culminating in today's smart sensors or "Sensor 4.0". This paper briefly reviews the development of sensor technology over the last 2 centuries, highlights some of the potential that can be achieved with smart sensors and data evaluation, and discusses success requirements for future developments. In addition to magnetic sensor technologies which allow self-test and self-calibration and can contribute to many applications due to their wide spectrum of measured quantities, the paper discusses condition monitoring as a primary paradigm for introducing smart sensors and data analysis in manufacturing processes based on two projects performed in our group.
\end{abstract}

\section{Introduction}

"Industrie 4.0", branded the fourth industrial revolution, is in fact more of a political vision than a new technical paradigm (see Plattform Industrie 4.0, 2018; Hightech-Strategie: Industrie 4.0, 2018; Dossier: Digitale Transformation in der Industrie, 2018): it is simply the continuing progression of achieving better knowledge and control over the entire production process that has been ongoing since industrialization made efficient mass production possible. The main benefit of this new way of looking at things is the chance to establish new business models. This is actually expressed better by the Anglo-Saxon term Industrial Internet of Things or IIot (Industrial Internet Consortium, 2018) because it hints at transferring successful business models of the new economy to industrial application; even more indicative are terms like digitalization or, short and pithy, Googlification. In fact, other application fields especially in consumer services are far ahead of industrial production processes in making use of the power of digitalization. Note that this is actually one fun- damental difference between the common approach and the new thinking: services and hence the value to the customer are more important than products.

This paper addresses the importance of sensors, instrumentation, and measurement science for Industry 4.0 and discusses potential and trends; it is based on two conference presentations addressing smart sensors, their relevance for Industry 4.0, as well as the requirement for an expanded uncertainty evaluation (Schütze, 2015; Schütze and Helwig, 2017); a shorter German version was recently published elsewhere (Schütze and Helwig, 2016). In the field of sensors and instrumentation the trend towards smart sensors has long been established in aspects like better performance, higher integration, and multi-parameter sensing, but also built-in intelligence as well as secure and safe networking (Gassmann and Kottmann, 2002; Sensor-Trends, 2014). Intelligent sensor systems allow e.g. self-identification or diagnosis up to self-configuration, calibration, and repair, often subsumed under the term self-X (Akmal Johar and König, 2011). In 
analogy to Industry 4.0 the term Sensor 4.0, coined by Peter Krause, the chairman of AMA e.V. (AMA, 2018), snappily designates the current development in sensors and measurement science. Similar to the four phases of industrial development, this classification discriminates between purely mechanical indicators (e.g. the aneroid barometer, also referred to as a Vidie can, invented by the French physicist L. Vidie in 1844), electrical sensors (e.g. classic strain gauges invented simultaneously by E. E. Simmons at Caltech and A. C. Ruge at MIT in 1937/38), the state-of-the-art electronic sensors (e.g. electronically compensated pressure sensors available since approx. 1970), and, finally, smart sensors; cf. Fig. 1. This overview also shows how strongly the industrial evolution is connected to sensors and instrumentation. Note that the importance of sensors is not limited to industrial processes, but is in fact strongly influencing all current megatrends like smart cities or smart mobility. The best examples of highly integrated sensor platforms are in fact smartphones which typically integrate around 15 different sensors and heavily make use of multisensory signal evaluation, e.g. for navigation which relies on accelerometers, gyroscopes, magnetometers, and pressure sensors. At the same time these sensors are also used for other services like weather monitoring, screen orientation, step counting, and, last but not least, gaming. In this case, the sensors are in fact "dumb" sensors, with the integration and data fusion between different sensors achieving a smart platform.

The relevance of modern sensors and instrumentation is also reflected by the economic data which show a continuous growth of more than $6 \%$ CAGR in turnover from 2005 to 2015 and a steady increase in jobs of almost $40 \%$ over the same period, compared to stagnation in the industry as a whole (based on an analysis by AMA). The companies in this field also invest an average of $10 \%$ of their turnover in research and development and are therefore attractive employers for young engineers and physicists.

\section{State-of-the-art and current trends}

A current trend in sensor technologies is the use of active measurement principles that are used in various sensor elements and systems. Examples are magnetic sensors, e.g. Hall sensors using spinning current (Munter, 1990), internal calibration and even correction of offset temperature coefficients through integration of internal chip heaters (StahlOffergeld, 2011), MR sensors using the compensation principle to suppress temperature cross-sensitivity (Marien and Schütze, 2009), micromechanical accelerometers (also using compensation or internal calibration methods) and gyroscopes (using the Coriolis effect with active vibration excitation), and Coriolis-based flow sensors or chemical sensors using temperature modulation for improved selectivity, sensitivity, and stability (Reimann and Schütze, 2014; Baur et al., 2015). Active modes of operation also offer additional potential for self-diagnosis, which is already extensively being used in automotive applications (Ochs, 2013). This does not only apply to inertial sensors, where the correct function is checked with internal excitation, but also to e.g. the lambda probe: here the time constant for heating to the desired operating temperature is used to detect faults, e.g. cracks of the ceramic. Self-diagnosis is especially important for applications in safety and security. Fire detection and explosion protection could not be addressed with low-cost gas sensors, which are prone to poisoning. Here, dynamic operation also allows detection of sensor faults, e.g. poisoning of the sensor material (Bastuck et al., 2015; Schüler et al., 2015).

Magnetic sensors are especially suitable for self-X methods as an internal calibration can be realized by coils integrated in the system or directly on the chip. Furthermore, as the sensors are very small and integrated on silicon chips with good thermal conductance, heating of the sensors is also possible, thus allowing direct determination of thermal cross-sensitivity. Many principles are today already implemented in integrated Hall sensors (e.g. 3-D-HallinOne sensors developed by Fraunhofer IIS), due to their being based on standard CMOS technology, thus allowing simple integration with analogue and digital electronics (Stahl-Offergeld, 2011). MR sensors are not yet as advanced due to the difference in technologies for sensor chips and electronics, but the potential for self-X technologies is increasingly being studied (Akmal Johar and König, 2011; MoSeS-Pro, 2015). Note that future trends might include internal traceability of the sensor function by making use of quantum standards for SI units so that sensors might be truly calibrated during operation as proposed by Kitching et al. (2016) (see also NIST-ona-Chip, 2018).

Integrated Hall sensors can serve as one specific example highlighting the potential of (magnetic) sensors and their integration with advanced modes of operation and data treatment in the sensor itself. Hall sensors are used in many applications and are sold in large quantities at surprisingly low cost considering their performance. While on the outside these sensors still resemble the well-known simple Hall plate, a purely analogue, current, or voltage driven sensor with voltage output to measure the magnetic field, they are much more complicated inside. The spinning current principle, periodically switching driving and output contacts, has already been used for a long time (Munter, 1990) to compensate for various unwanted aspects (unsymmetrical geometry, variations in doping of the Hall layer, mechanical strain, and temperature differences), which would otherwise result in large offsets and therefore reduced resolution (StahlOffergeld, 2011). Even after spinning current compensation which is achieved by typically four measurements with current induced in all four directions of the Hall plate and subsequent averaging of the results, a residual, temperaturedependent offset remains. By integrating a small excitation coil directly on the chip this offset can be determined during normal operation. In addition, a small heater can also be 


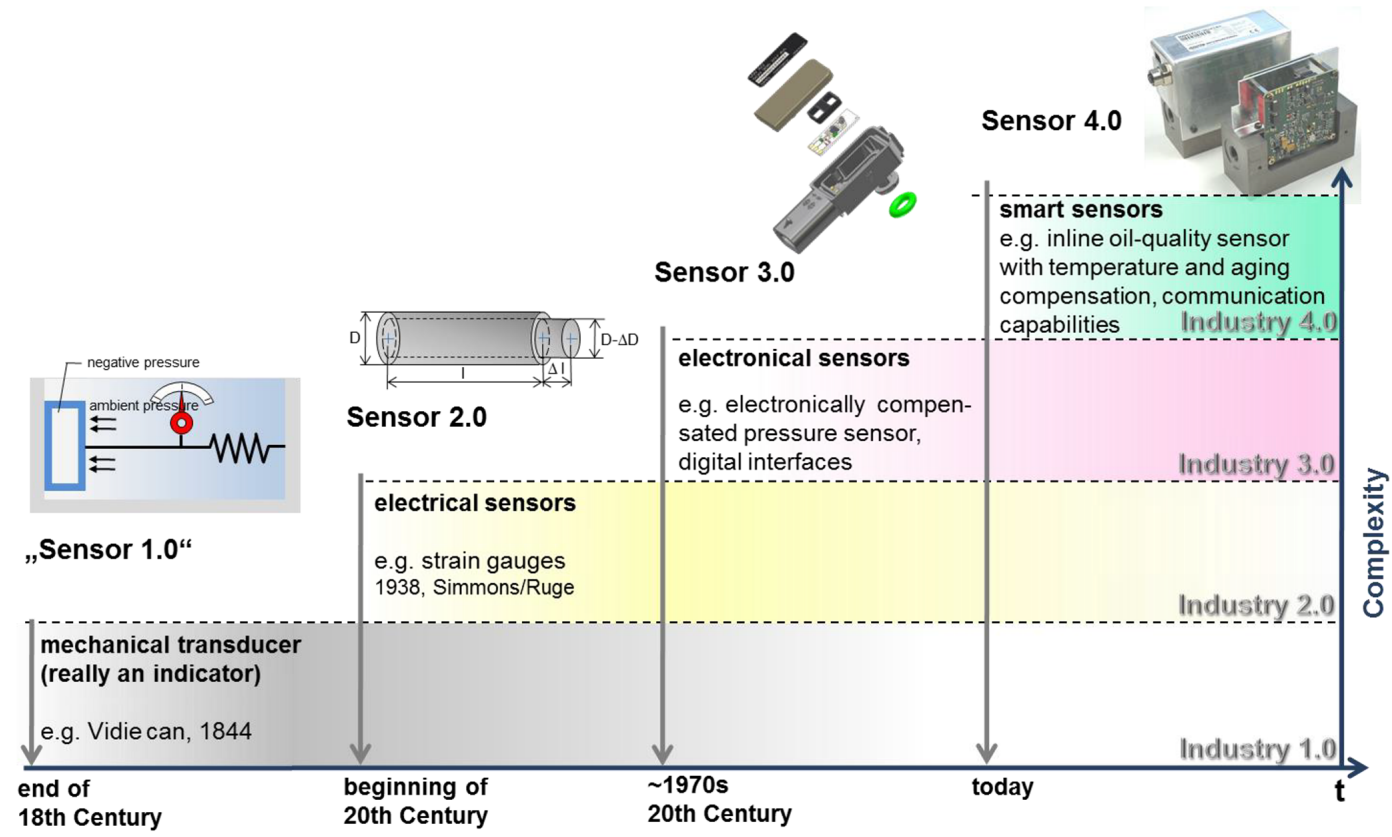

Figure 1. Historic evolution from "Sensor 1.0" (without electrical output this is not a sensor according to the usual definition) to smart sensors, i.e. "Sensor 4.0" (based on Peter Krause, chairman of AMA e.V.).

integrated on the chip, which can induce small temperature changes to determine the temperature coefficient (TC) of the offset, thus allowing a digital correction of the temperaturedependent offset with a linear model. To achieve offset compensation over a wide operating temperature range, this procedure can be repeated at different ambient temperatures. The resulting residual offset and thus the effective resolution of the sensor is greatly improved by this procedure by more than 1 order of magnitude from $\pm 1 \mathrm{mT}$ down to $\pm 50 \mu \mathrm{T}$ (Stahl-Offergeld et al., 2009; Stahl-Offergeld, 2011). However, due to cost restraints the sensor only contains one AD converter, which means that chip temperature and Hall voltage cannot be measured simultaneously. To avoid errors in the determination of the offset TC the thermal behaviour of the chip is therefore modelled with several time constants. Furthermore, several measurements during this self-calibration and a statistical evaluation of the resulting variations are used to check whether the applied external field has changed during this procedure, which could lead to false values for offset and offset TC; for further details, the reader is referred to Stahl-Offergeld et al. (2009). In addition, other production-related parameters of the sensor chip can be determined with a suitable strategy combining on-chip measurements and digital evaluation (Stahl-Offergeld et al., 2010; Stahl-Offergeld, 2011). This example shows that even a seemingly simple Hall sensor today determines the required measurement value based on a complex digital process. Note that a strict determination of the resulting measurement uncertainty based on the GUM principles (GUM, 2008) would result in a very complex process if the complete system and a full physical model were to be considered. In this case, a suitable statistical approach for determination of the uncertainty would seem more suitable.

Thus, smart sensors with additional functionality provide a significant added value for higher-level functions, e.g. in production systems. The correct sensor function is also required for the condition monitoring of complex systems (see Sect. 3 below). In this case, the correlation of sensor data within the system can also be used to verify the correct sensor function; however, in this case, the sensor fault diagnosis has to be performed on a higher level within the system.

Additional trends that will be initiated or at least pushed further by the Industry 4.0 paradigm are the following.

- Measurement as a service: this could be a trend similar to the service provided by Uber in public transport, i.e. measurement services or even individual results are sold instead of instruments. Note that the measurement uncertainty - determined online by self-calibration - will then influence the price.

- Traceability of individual components down to screws, individual gears and even gaskets: this additional knowledge will allow tolerance measurement in the assembly of (sub-)systems and is also required for a comprehensive condition monitoring to assess the influence of individual processing steps and machines on the final result.

- Self-learning systems: the correlation between sensor data as well as other process and ambient parameters 
can be evaluated to ensure the correct function of the system in the sense of a system self-diagnosis by making use of machine learning (Cachay and Abele, 2012). So far it is unclear whether unsupervised methods are sufficient or whether supervised learning, see Sect. 3 below, is required, i.e. knowledge of the current system status for training the evaluation.

- Semantic technologies for analysis of complex systems: interpretation of measurement values beyond the purely data-based approaches could offer further opportunities, e.g. for plausibility checks of sensor data and for providing confidence values for (fault) causes. Note that the World Wide Web consortium (W3C) started working on a semantic sensor network ontology as early as 2005 which allows representation of measurement values and their significance (Semantic Sensor Network Ontology, 2017).

The last example shows that the importance of sensors and measurement technology was recognized also by other parties, which leads to some parallel and independent developments. Interestingly, however, aspects like measurement uncertainty and sensor self-monitoring are not addressed in the context of semantic technologies even though semantic representation would be highly valuable especially for these aspects.

\section{New measurement paradigm: condition monitoring using data-based modelling}

The potential of data-based sensor signal evaluation is demonstrated by the iCM Hydraulics project (2013). In this project a hydraulic model system combining a primary circuit with variable load and a secondary circuit for cooling and filtration were used to study the identification of typical system faults (internal pump leakage, delayed valve switching, pressure leakage in the accumulator, reduced cooling efficiency) only based on an evaluation of the usual process sensors (pressure flow rate, temperature, electrical power).

Figure 2 provides an overview of the approach: the hydraulic system is equipped with a total of 17 physical and virtual (e.g. efficiency calculated from electrical power input and hydraulic power output) sensors, which are read out with up to $100 \mathrm{~Hz}$. The system was used to simulate a periodic industrial process with a work cycle of 1 min duration. In each cycle a total of approx. 50000 raw values is recorded, which are interpreted as a high-dimensional measurement vector. A multi-step dimensionality reduction covering signal pre-processing, feature extraction, and selection yields a projection obtained by linear discriminant analysis (LDA) (Duda et al., 2000), which allows classification of the system status, i.e. identification and quantification of the fault. Classification can be performed with various methods, e.g. k-nearest neighbours, support vector machines (SVMs), or artificial neural networks (ANNs). Note that pre-processing and feature extraction are realized with unsupervised methods, i.e. without making use of the system status, while feature selection - here based primarily on Pearson correlation of features and fault status - and LDA projection are supervised methods, i.e. require the knowledge of the system status (Helwig and Schütze, 2014). The evaluation is based on a comprehensive training phase in which all combinations of all fault states are measured. The complete training is based on several thousand working cycles and requires approx. 3 days, primarily due to the relatively slow equilibration of the temperature after changing the cooling efficiency. The complete training data set contains almost 120 million raw data points. A systematic validation, e.g. based on k-fold cross-validation, completes the development of the statistical model and ensures that no overfitting occurs in spite of the high-dimensional input data set and the supervised training methods (Helwig and Schütze, 2014).

In this example statistical methods were primarily used for feature extraction. The working cycle was divided into 13 sections (complete cycle and 12 sections representing different constant or changing pressure levels, respectively) and the first four statistical moments (mean, standard deviation, skewness, and kurtosis) were determined for each sensor in each section. This can be implemented on low-cost hardware very efficiently, but is still the computationally most costly step of the training procedure. This step requires a few minutes on a standard PC for the complete data set with several 1000 cycles. The resulting almost 900 features (17 sensors $\cdot 13$ sections $\cdot 4$ statistical moments) result in a feature space that still has too many dimensions for efficient classification. Therefore, feature selection based on correlation between features and target classes, i.e. fault level, is used which is computationally extremely efficient, same as the calculation of the LDA projection to obtain the 2-D plots, $\mathrm{cf}$. Fig. 3, or ideally only one discriminant function (DF) per system fault. These two computation steps only require fractions of a second. Even faster is the classification of a new working cycle, i.e. extraction of the selected features, projection in the LDA space for each system fault, and classification based on a k-nearest neighbour classifier, which can thus be performed in real time even on a low-cost microcontroller-based system.

The performance of the approach is shown in Fig. 3 for the four studied system faults: each fault state can be identified independently and its severity or level can be estimated with surprisingly high accuracy. The cooler efficiency, for example, can be estimated with better than $10 \%$ (the reduced cooler efficiency was simulated with pulse width modulation of the power supply, and the percentage gives the duty cycle used); the accumulator pressure can be determined with an uncertainty of approx. 5 bar. Projected test data which were not used to build the model (open symbols) show that the model allows correct classification of unknown states and even that an extrapolation of data outside the training range is possible within limits. 

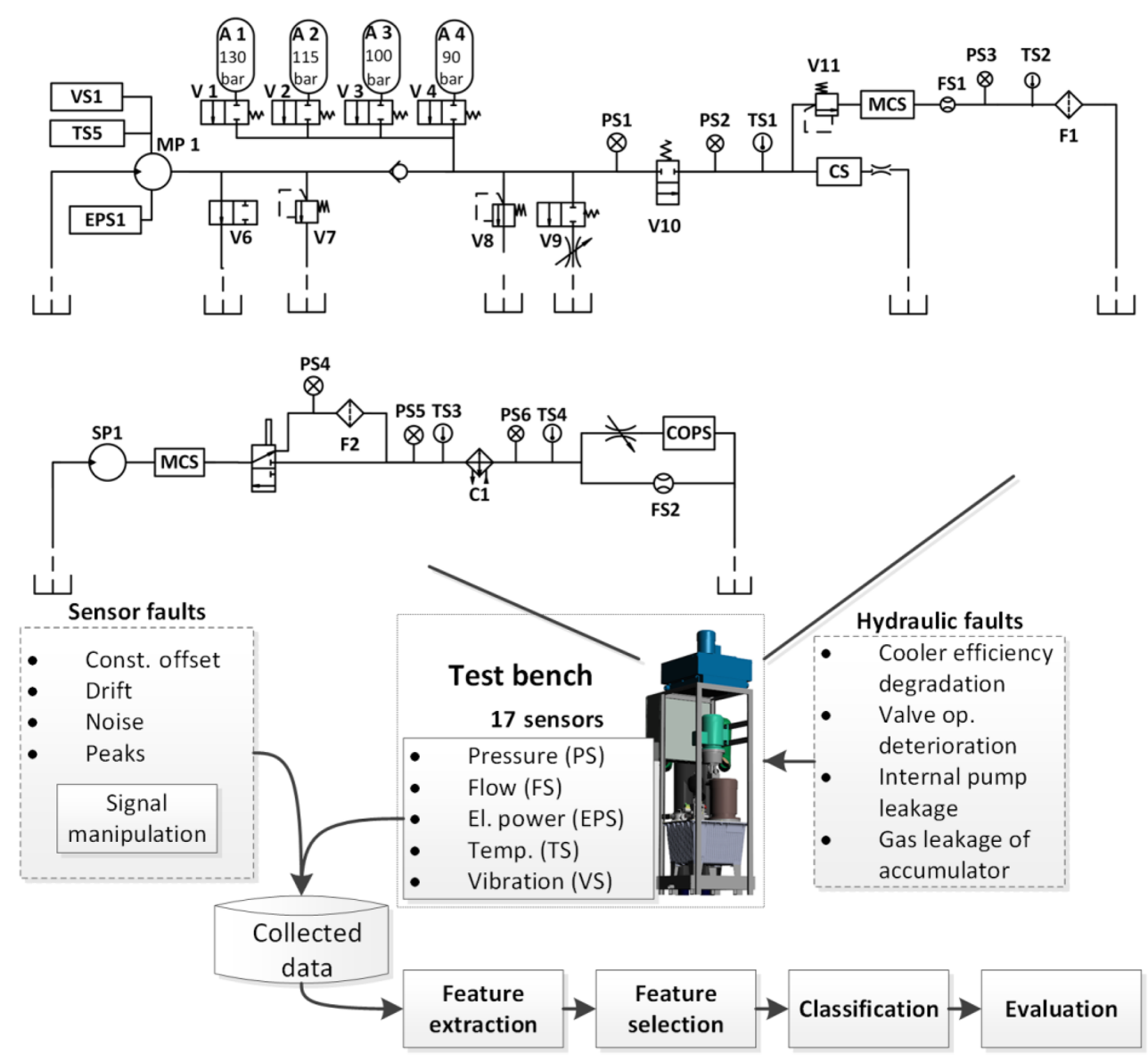

Figure 2. Hydraulic system and concept for the data analysis within the iCM-Hydraulics project (Helwig and Schütze, 2015). A statistical model was built allowing for independent identification of various hydraulic system faults as well as identification of sensor defects. System condition monitoring remained possible even with sensor faults by excluding the identified faulty sensors from the analysis.

In further experiments we have shown that the training can be transferred from one system to a second, identical system after some calibration, i.e. shift of the LDA projections for the correct system state (Helwig et al., 2015a). Given the high performance which was not expected when designing the experiments, we also studied how sensor faults would influence the classification results. For this, sensor offset, drift, noise and signal drop-outs were simulated in the recorded data for all sensor channels and the resulting data were used to classify the system state. Not surprisingly, the classification rate is drastically reduced, especially for monitoring of pump leakage and the hydraulic accumulator. To allow automatic recognition of sensor faults, these were defined as new targets for the classification algorithm and trained using the same completely automated approach. Again, the simulated sensor faults could be recognized with high reliability independent of the system state as shown in Fig. 4 for two exemplary sensor faults. In fact, sensor faults can be diagnosed before they lead to false classification of the system state (Helwig and Schütze, 2015). Correct classification of the overall system state is still possible by excluding the de- fective sensor(s) from the evaluation and making use of the remaining sensors. In fact, up to five of the most important sensors can be excluded from the evaluation and still a correct classification rate of more than $80 \%$ is achieved (Helwig and Schütze, 2015).

The projection shown in Fig. 4a can also be presented in a different way, as the second discriminant function (DF2) obviously does not provide relevant information for the offset classification. Plotting the data as a histogram results in the plot shown in Fig. 5 which shows nearly normal distributions for all six classes with a constant FWHM (full width at half maximum) or standard deviation. Thus, this projection could be used not only to determine or "measure" the sensor offset; it also provides an estimate for the uncertainty with which this offset can be determined, considering only type A uncertainties. Note that this also holds for the two classes with 2 and 10 bar offset, which were not used for calculating the LDA, i.e. building the statistical model.

Similarly, the histogram for the accumulator pressure shown in Fig. 6 also yields a constant standard deviation, which increases with increasing temperature range, thus in- 

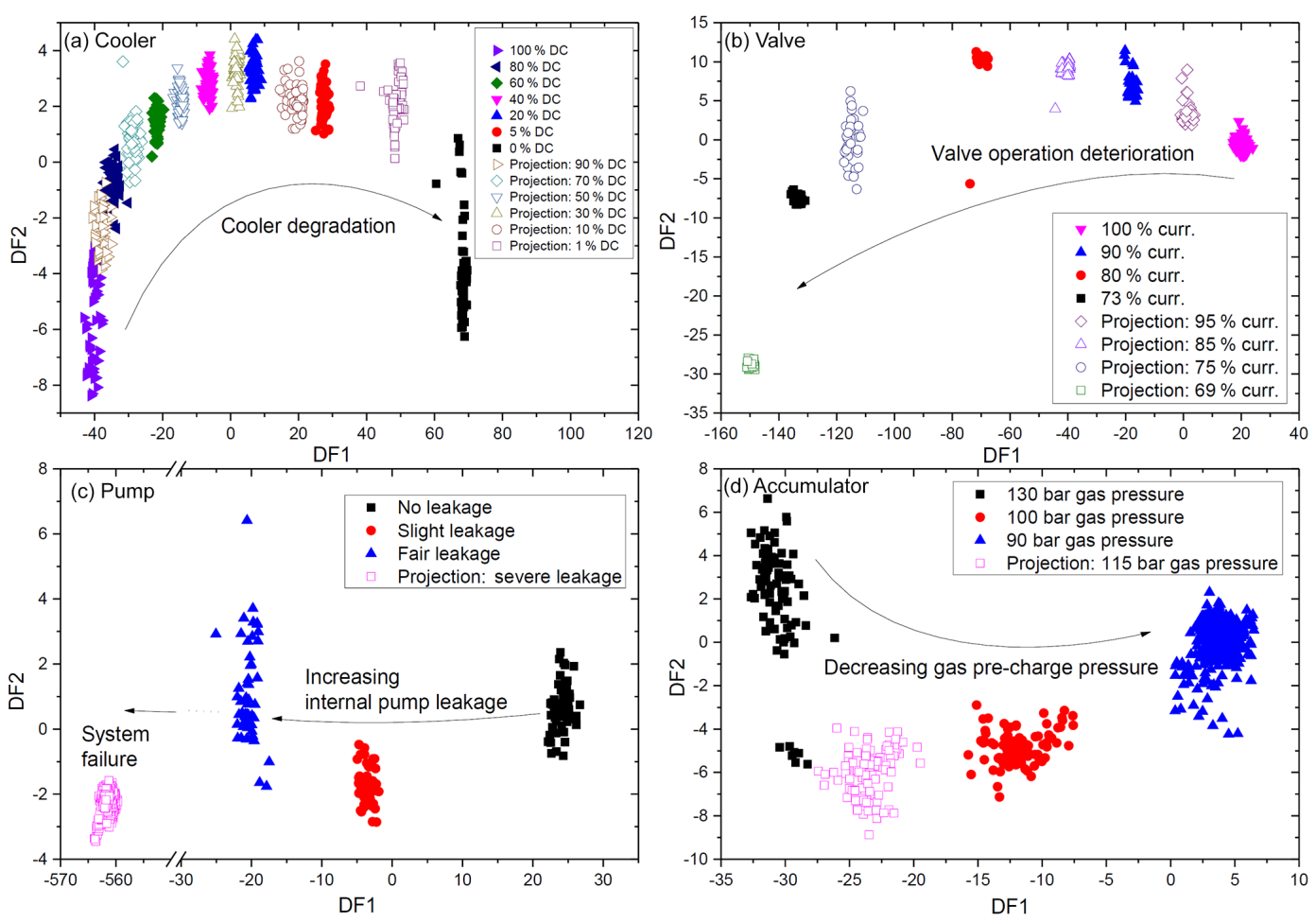

Figure 3. Results for determination of the four system faults studied: cooler degradation (a), valve operation (b), internal pump leakage (c), and accumulator pressure (d). Full symbols show data used for determining the statistical model, and open symbols show additional test data not used in the training which prove that unknown data are interpreted correctly (Helwig and Schütze, 2015).

dicating a measurement uncertainty depending on ambient conditions, which is quite common for many sensors. This also shows that the performance of the condition monitoring approach deteriorates drastically when the temperature of the system, or more specifically the temperature of the hydraulic oil and with it the viscosity, changes over a wide range. While the accumulator pressure can be estimated with an uncertainty of less than \pm 5 bar for a temperature range of $10^{\circ} \mathrm{C}$, an increase in the temperature range to $20^{\circ} \mathrm{C}$ leads to significant overlap between the different classes with an uncertainty of at least \pm 10 bar. Note that narrowing the temperature range further does not reduce the uncertainty correspondingly, probably due to noise of the sensor data contributing to this result (remember that an discriminant function is a weighted sum of different features, i.e. sensor values). To take this effect into account the training of the statistical model would either need to be extended to include data over a wide (oil) temperature range or the exact interpretation of the system condition can only be done in a typical operating window. The latter approach is surely better suited for typical industrial applications, especially as a full condition monitoring is not required with high temporal resolution, i.e. for classification of wear processes, due to the normally slow progression of the system deterioration. On the other hand, if this approach were to be used for mobile (hydraulic) machinery, i.e. loaders, the ambient and also the operating tempera- tures would depend drastically on location and weather conditions. In this case, either an expanded training over the full operating temperature range would be required or perhaps several different projections selected based on the relevant temperature level. In any case, training effort would increase to allow universal condition monitoring.

The examples shown here clearly demonstrate the potential of data-based statistical modelling for condition monitoring of complex systems purely based on existing process sensors. Thus, a cost-efficient and powerful monitoring can be achieved which allows interpretation of the results also in terms of the measurement uncertainty of the systems status, i.e. the uncertainty is nearly constant over the full range from a system in mint condition to near failure, but the uncertainty increases if additional factors, in this case significant changes in the oil temperature, have to be taken into account. Note, however, that this does not apply to all system faults. In this example a varying uncertainty was observed for the valve switching behaviour which increased over the monitored range, which might be due to a non-linear relation between features and resulting discriminant function and the fault status. Even more problematic is the observation that the variation of results for test data does not show a normal distribution, i.e. a simple interpretation of the standard deviation as measurement uncertainty is not possible, and, 

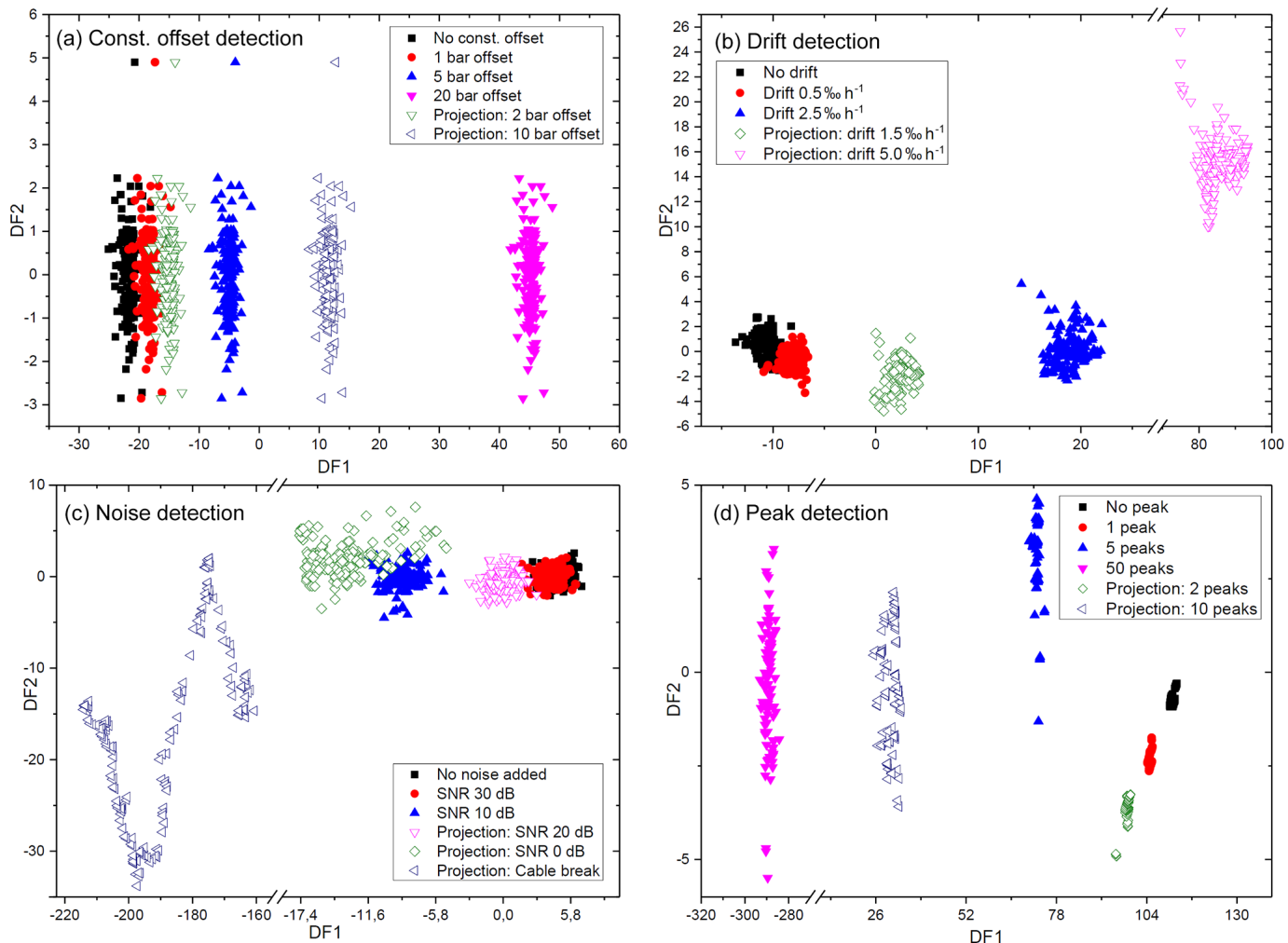

Figure 4. Results for identification of sensor faults offset (a), drift (b), noise (c) and peaks (signal drop-outs, d). Full symbols show data used in determining the model for the sensor faults diagnosis, and open symbols show additional data not used in the training, which again proves that unknown data are interpreted correctly (Helwig and Schütze, 2015).

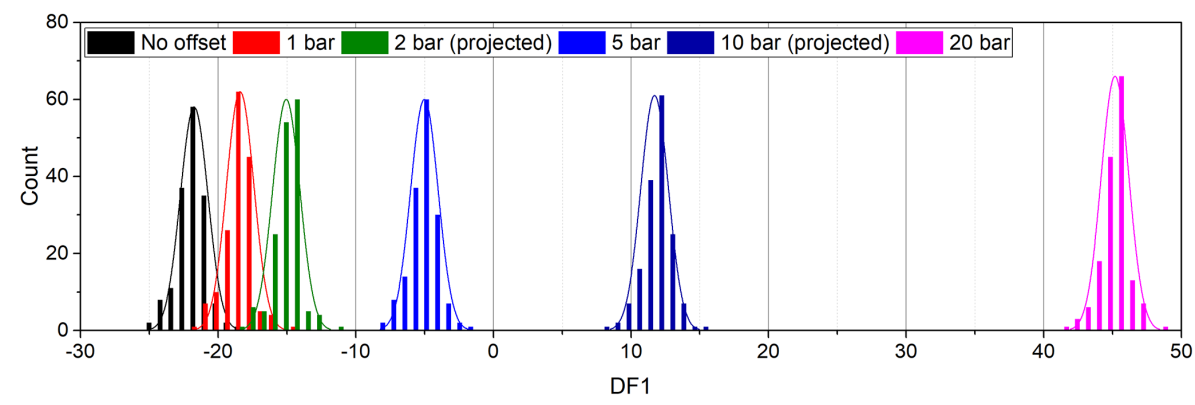

Figure 5. Plotting the data from the LDA analysis as a histogram of the first discriminant function (DF) shows that this projection results in a linear relationship allowing determination of the sensor offset. An estimate of the uncertainty for the offset is also possible due to the constant standard deviation of the data; note that this includes data at 2 and 10 bar offset, which were not used for building the statistical model.

furthermore, the interpretation of the statistical results as a "measurement" of the system state might not be justified.

\section{A modular approach for smart sensor networks and condition monitoring}

The successful preliminary work in iCM Hydraulics resulted in the establishment of a successor project, in which the developed methods are transferred to an open sensor system toolbox. In this project (MoSeS-Pro, 2015) magnetoresistive sensors (AMR, GMR, and especially TMR) are primarily used to measure current, position, and angle, but other (micro)sensors, e.g. MEMS sensors for vibration, pressure, or thermal radiation, are also used to extend the measurement spectrum. These sensors are also integrated into components and subsystems (Helwig et al., 2017b) to allow improved performance and condition monitoring, both as an end-of-line test in their production and during their opera- 


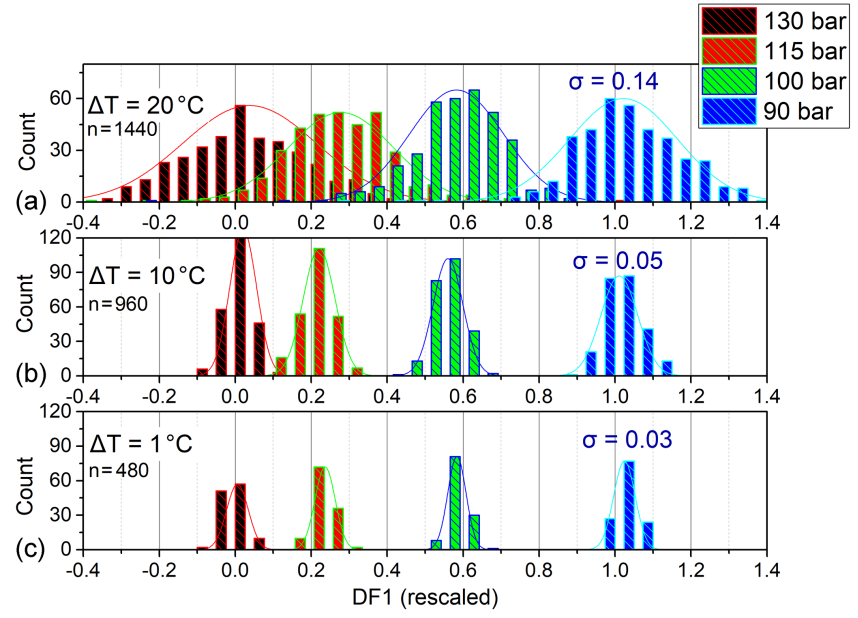

Figure 6. Histogram of the sensor data/features for classification of the accumulator pressure vs. first discriminant function. Here, the data were rescaled (compare Fig. 3d) to centre values for 130 bar (nominal pressure) at 0 and data for 90 bar at 1 . The standard deviation for small temperature range (c) seems to be determined by noise from the sensors/features, while it is strongly influenced by variations of the oil temperature for increasing temperature range (a).

tion in manufacturing systems. In this project, modular electronics and software algorithms are developed, allowing the required signal pre-processing and feature extraction directly in the smart sensor. Otherwise, signals recorded at high frequencies of several $100 \mathrm{MHz}$ would result in data rates which would overload the higher levels. In addition, novel self-X methods, wireless sensor interfaces, and energy harvesting are developed for easy integration and initialization of system operation. Figure 7 gives an overview of the modular approach.

As shown above, statistical data analysis is a powerful tool for condition and process assessment without firm and detailed expert knowledge since most of the underlying algorithms are self-optimizing and can be concentrated in automated signal processing chains. However, this approach, especially in the case of supervised learning, requires a sufficient quality of training data, i.e. typically cyclical processsynchronized sensor data which are annotated with corresponding classes, i.e. the target vector for which the statistical model is to be trained. The typical steps for offline analysis (Fig. 8a) are signal pre-processing, feature extraction, and selection as well as classification with subsequent evaluation and can be interpreted as a gradual dimensionality reduction. Feature extraction and selection can be fully automatized using a modular approach based on complementary algorithms to extract information from the time domain, i.e. with adaptive linear approximation (ALA), from the frequency domain, i.e. with Fourier analysis, from the timefrequency domain, i.e. using wavelet analysis, or the overall system, i.e. based on principal component analysis (PCA). Similarly, complementary techniques are used to select suit- able features and feature combinations, i.e. simple correlation analysis or recursive feature elimination support vector machines (RFESVMs) for linear or RELIEFF for non-linear separability (Schneider et al., 2017). In this way, the signal processing software as part of the sensor kit is realized in a highly modular design since heterogeneous sensors differ significantly regarding signal shape, time and spatial resolution, and target information to be extracted.

An example of the application of this toolbox is shown in Figs. 9 and 10. A miniaturized sensor system prototype was designed for integration in an electromechanical cylinder (EMC). These are increasingly applied as feed drives in machine tools, due to their unique combination of high loads, precision, and flexibility. The sensor system contains a range of (partially redundant) sensors (linear and rotary encoders, 3-D accelerometers, microphone, temperature and IR radiation sensors). Currently, the sensor prototype consists of two separate subsystems: first, two stacked sensor printed circuit boards (PCBs) (Fig. 9) mounted on the front surface of the ball screw inside the EMC housing (Festo ESBF-BS-63-400$5 \mathrm{P}, \varnothing 63 \mathrm{~mm}, 400 \mathrm{~mm}$ stroke, $5 \mathrm{~mm}$ spindle pitch, axial load max. $7 \mathrm{kN}$ ) containing in total nine MEMS sensors. Furthermore, the rotary position of the spindle shaft is measured by an AMR Wheatstone bridge sensor (Doms and Slatter, 2014) with external bias magnet generating the support field which interacts with ferromagnetic teeth of the spindle shaft. This sensor is positioned at a fixed position in the cylinder housing close to the ball bearing (cf. Fig. 1a) pointing to the thread with a working distance of $1 \mathrm{~mm}$. During rotation, the relative position of sensor and teeth changes, periodically resulting in sine and cosine sensor signals.

To evaluate the sensor system in a condition monitoring scenario, we induced a local abrasion of the spindle at stroke position $185 \mathrm{~mm}$ and recorded several stroke movements with varying velocity and three repetitions. For signal processing, short-time Fourier transform (STFT) was applied (length 10000 /overlap 2000 samples) with subsequent feature extraction and selection as previously demonstrated (Helwig et al., 2015b). Feature extraction captures a total of 210 statistical parameters such as median, variance, skewness, and kurtosis in different intervals of the amplitude spectra of three acceleration axes. The features are selected by $F$-value ranking of univariate ANOVA and dimensionally reduced to three discriminant functions (DFs) using LDA to obtain the maximum class separation. The latter algorithms are supervised learning methods, i.e. require class-annotated data which were given as velocity information and a local spindle condition traversed by the spindle nut. Figure 10a shows the resulting 3-D projection of sensor data with the planes DF1-DF2 and DF1-DF3 separating the different velocity levels and spindle conditions, respectively. Here, the velocity classes with 10,20 , and $50 \mathrm{~mm} \mathrm{~s}^{-1}$, respectively, were used for training and the class with $30 \mathrm{~mm} \mathrm{~s}^{-1}$ velocity was used for evaluation. The intermediate velocity class fits well into the data-based model and the fault identification 


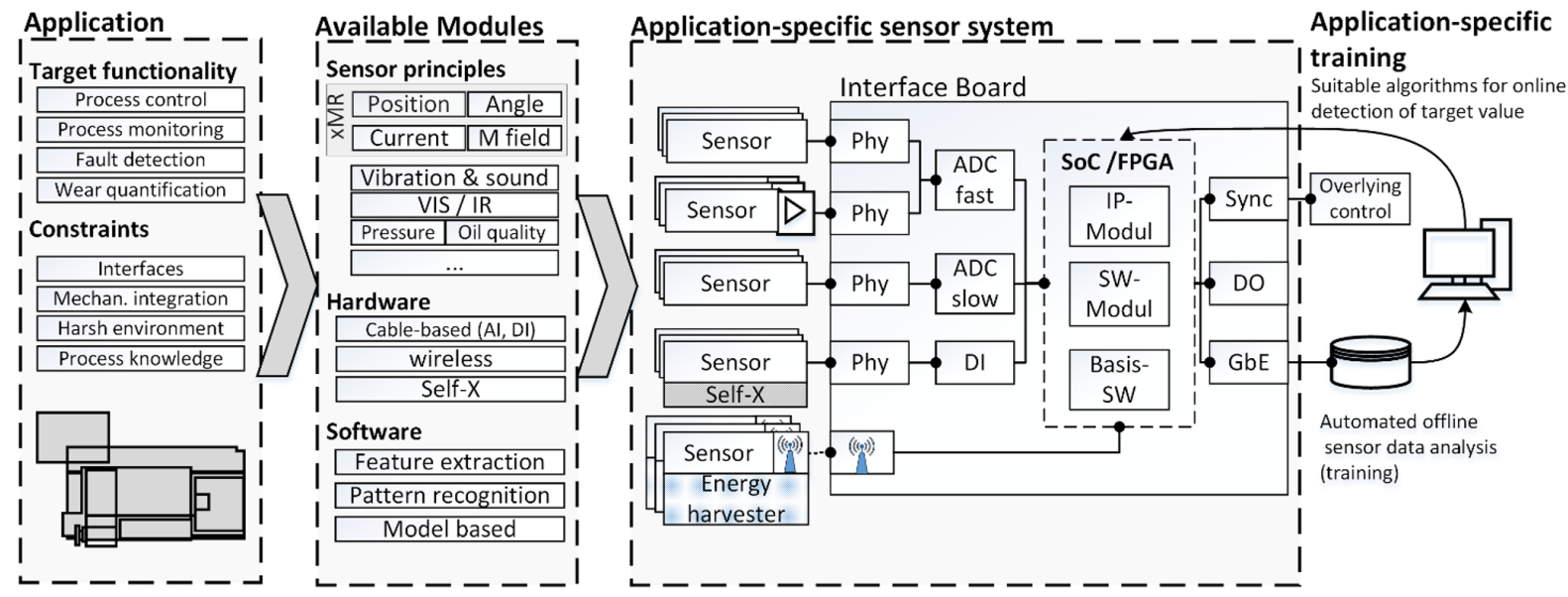

Figure 7. Application-specific design of the modular MoSeS-Pro sensor system kit combining various (micro-)sensors, especially xMR sensors, but also MEMS vibration, sound, pressure or IR radiation sensors, with electronics for data acquisition and pre-processing as well as communication interfaces in application-specific sensor systems (Helwig et al., 2017a).

(a) Overall scheme
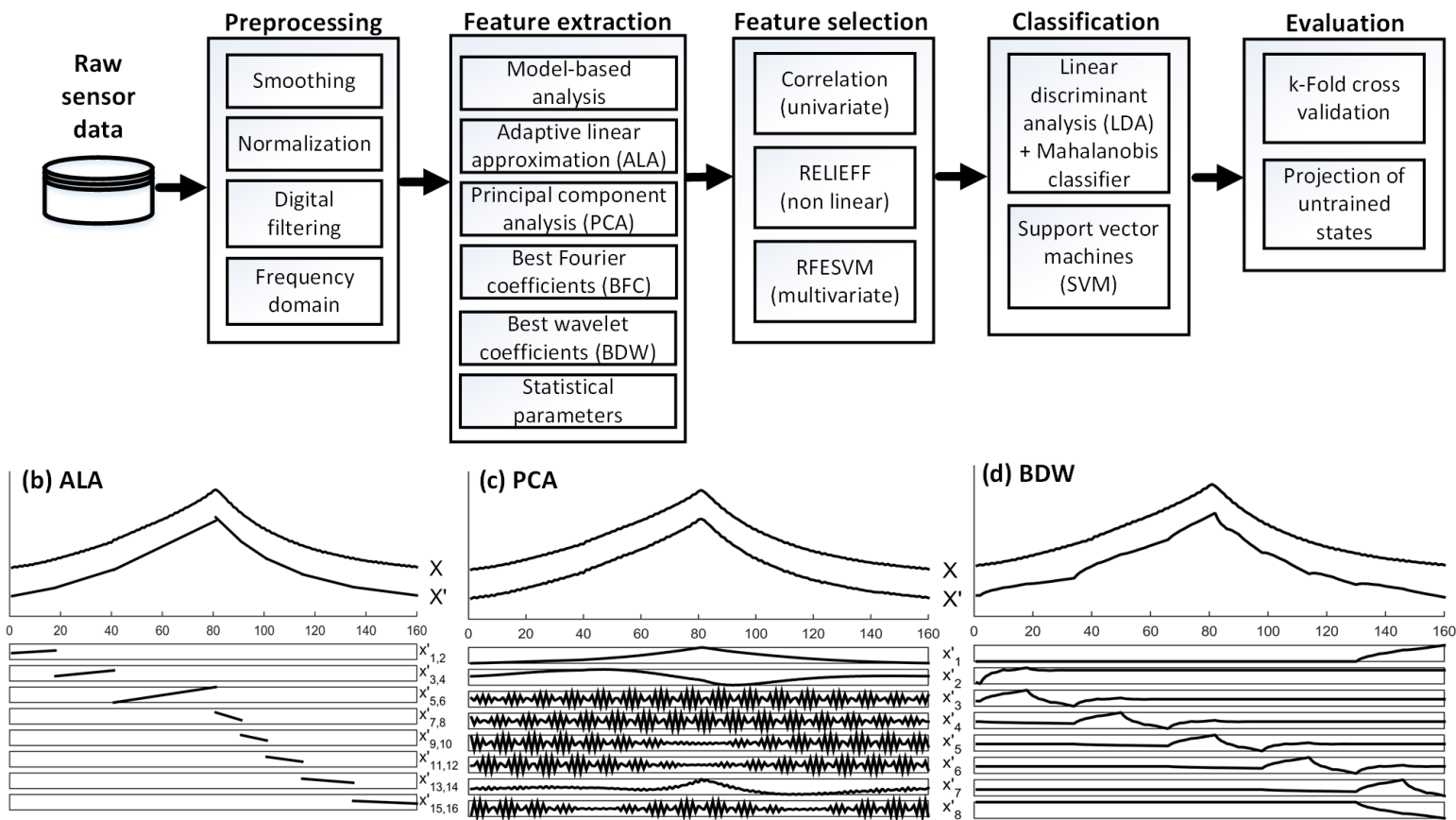

(c) PCA

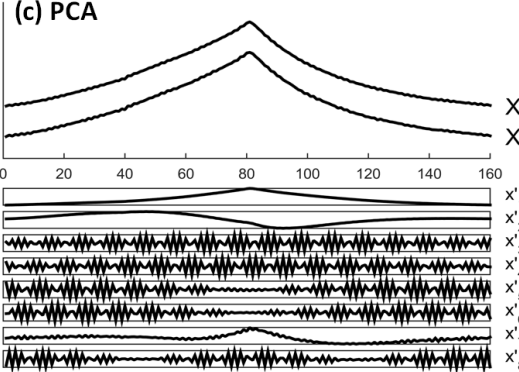

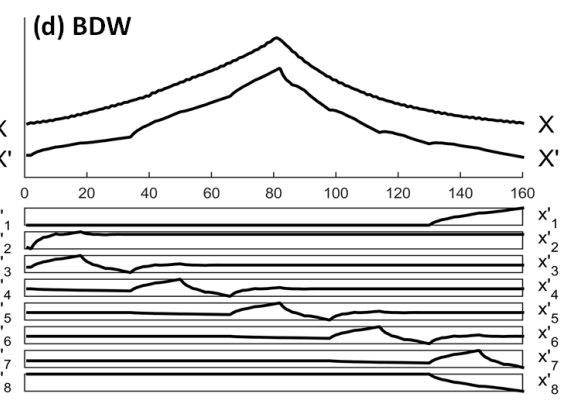

Figure 8. (a) Typical steps for offline data analysis and approximation of an exemplary sensor signal with different complimentary feature extraction methods: (b) adaptive linear approximation, (c) principal component analysis and (d) best wavelet coefficients using the largest Daubechies-4 wavelet coefficients (BDW: best Daubechies wavelet); in each case, $\mathrm{X}$ shows the original signal and $\mathrm{X}^{\prime}$ the approximated signal using 16 (b) and 8 (c, d) features, respectively.

rate improves with increasing velocity. Figure $10 \mathrm{~b}$ shows the plot of DF3 over stroke position clearly indicating the defect. The maximum is blurred, first, due to the interaction of balls and spindle defect over a distance of $30 \mathrm{~mm}$ and, second, also results from the STFT temporal blur. Furthermore, especially at low speeds with accordingly higher local res- olution, two local maxima can be seen indicating the entry and exit points of the spindle nut passing over the defect. This example shows that the stroke position dependent analysis of signals can be used for fault diagnosis differentiating between local anomalies such as defects of the spindle and 
(a)
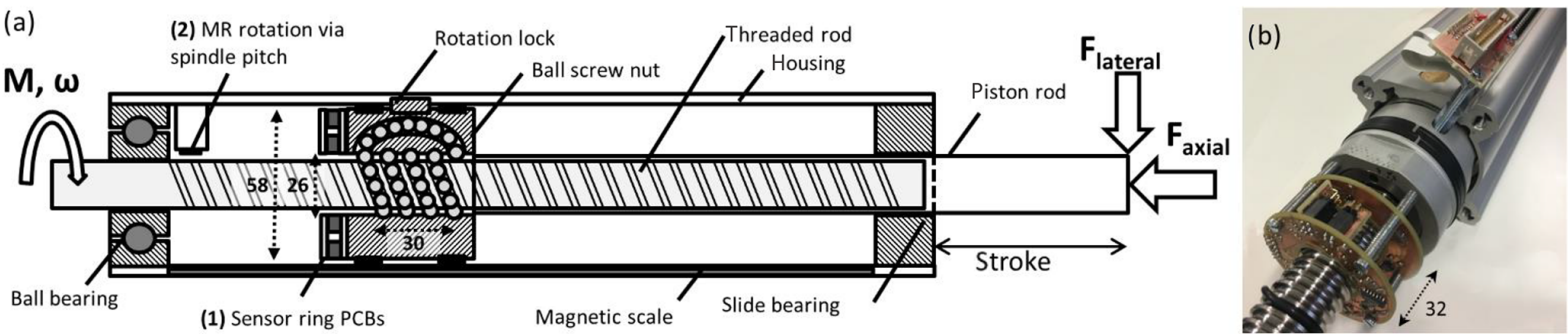

Figure 9. (a) Mechanical integration of the sensor system inside the EMC and (b) realization of the stacked sensor ring PCBs with the spindle shaft inside a disassembled cylinder (Helwig et al., 2017b).

global disturbances, e.g. of the ambient. For further details, the reader is referred to Helwig et al. (2017b).

To make full use of the MoSeS-Pro approach, data preprocessing and feature extraction need to be integrated into the sensor system to reduce the data load in the network and the cloud. However, this modular approach can also be used to design cost-efficient sensor systems for smart monitoring applications. In this case, a complete "over-instrumented" sensor set is used and the full sensor data are evaluated with the automated approach described above. Using the fairly simple and transparent algorithms allows identification of relevant sensors and features and, thus, the necessary acquisition bandwidth using an offline analysis. On this basis a greatly simplified sensor system can be defined for practical application. This approach would also allow us to choose an application-specific balance between sensor redundancy, i.e. to achieve robust operation as shown in Sect. 3, and cost efficiency.

\section{Conclusion and outlook}

Sensors and instrumentation are central driving forces for innovation, not only for Industry 4.0, but also for other megatrends that are described with the adjective smart, e.g. smart factory, smart production, smart mobility, smart home, or smart city. Intelligent decisions of complex systems are based on the knowledge of the system as well as ambient conditions and influence factors provided with high accuracy by sensors. The importance of sensors, measurement science, and smart evaluation for Industry 4.0 has been recognized and acknowledged by various authors (Imkamp et al., 2016; Sommer and Schütze, 2016; Walter, 2017) and has already led to the statement "Industry 4.0: nothing goes without sensor systems" ("Industrie 4.0: Ohne Sensorsysteme geht nichts") (Arnold, 2014). It should be acknowledged that notwithstanding all the euphoria and expectations for higher sensor production and sales volumes - especially when thinking about the Trillion sensor roadmap (Bryzek, 2013) - paradigm changes are expected, as is often the case in the digital revolution. Completely new business models like Uber and AirBnB already also exist in some sensor ap- plications. Today, Google already provides the best traffic data based on mobile phone data with much better actuality and precision than classic traffic monitoring based on dedicated sensors. In this application the network plays an important role and of course the amount of data: while individual movement data provide low quality, data fusion of a large number of movements provides the required information. Similar effects can in the future also be expected for environmental data, i.e. air quality, when gas sensors are integrated into smartphones in large numbers. The field of sensors and measurement science and especially the research community have to address this challenge to ensure that future standards are still set by GMA (VDI/VDE-Gesellschaft Mess- und Automatisierungstechnik), DKE (Deutsche Kommission Elektrotechnik Elektronik Informationstechnik), and AMA (in Germany), as well as BIPM, CEN/CENELEC and ISO worldwide, and not in Silicon Valley.

A possible approach for the sensor and measurement science community to play a bigger role in this development of Industry 4.0 might be the area of measurement uncertainty, which is simply not addressed by the computer science community today. In addition to making use of quantum standards integrated in smart sensors, an expanded view of the Guide for Expression of Uncertainty in Measurement (GUM, 2008) taking into account sensor data fusion and statistical modelling is highly desirable to make full use of the undisputed potentials and to continue with the success story of industrial production in high cost countries, which is one of the promises of Industry 4.0. Condition monitoring of complex production systems - from a single hydraulic press to a complete factory with assembly and test systems - can be one paradigm for the development of sensors and measurement science for Industry 4.0 as this immediately offers many economic advantages but can also be used for developing and testing new business models. A highly important aspect here is data security and with it the question of who owns which data and who has a right to access certain data. Consider a critical component being monitored in a complex production process: while the raw data are produced in the factory, the know-how for their interpretation lies with the component manufacturer. Forwarding complete process raw data to the 


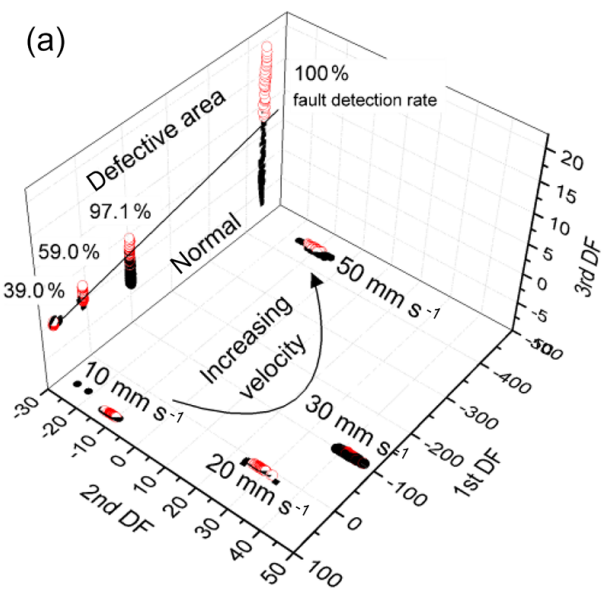

(b)

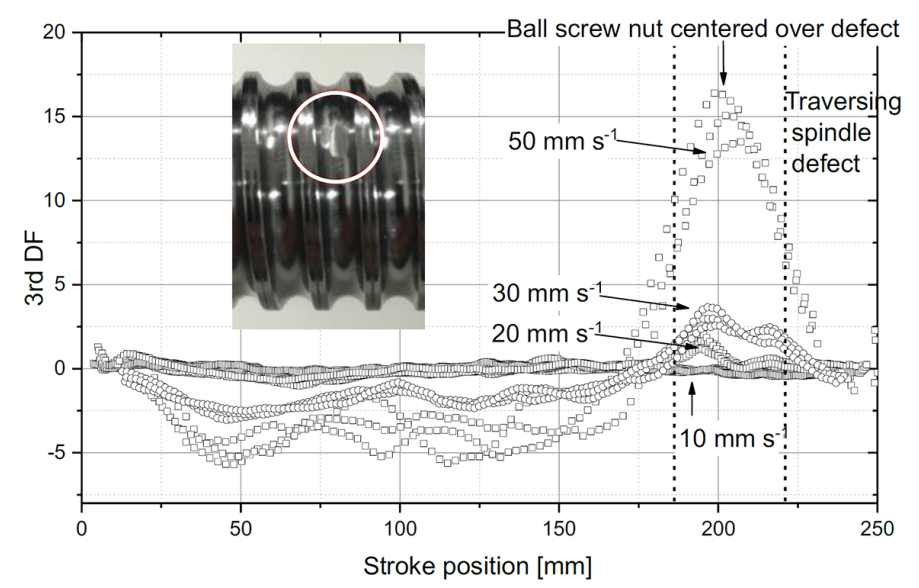

Figure 10. (a) LDA projection of 30 selected vibration features, $n=2883$, with training based on velocity classes 10,20 , and $50 \mathrm{~mm} \mathrm{~s}^{-1}$; classification rate determined for the Mahalanobis distance classifier with 10-fold cross-validation. (b) Deliberate abrasion as a local defect on the spindle and corresponding signal of DF3 vs. stroke position (moving average over 10 data points) (Helwig et al., 2017b).

component manufacturer is usually not an option, as this will also include confidential data, e.g. the production volume, from the factory. A successful business model will therefore require a certain level of trust between the involved partners but also a suitable abstraction level of the data from the component which would allow the required condition monitoring but no further insights into the confidential production process. This will of course be even more complicated if several component and sensor providers are involved to achieve the holistic condition monitoring approach based on data fusion. Perhaps this will lead to a new approach in data warehouse management with a novel type of neutral smart service provider to perform data anonymization and/or data analysis for all involved parties.

Data availability. The data are available in the UCI machine learning repository: https://archive.ics.uci.edu/ml/datasets/Condition+ monitoring+of+hydraulic+systems.

Author contributions. NH was responsible for the iCMHydraulics project and performed the measurements and evaluations presented in Sect. 3. NH (hardware) and TS (software) were jointly responsible for the modular condition monitoring (CM) toolbox developed within the MoSeS-Pro project and the results presented in Sect. 4. AS coordinated the research within both projects and wrote the manuscript.

Competing interests. Andreas Schütze is a member of the editorial board of the journal.
Special issue statement. This article is part of the special issue "Evaluating measurement data and uncertainty". It is not associated with a conference.

Acknowledgements. The iCM-Hydraulics project received funding through the EFI programme (support of development, research, and innovation in Saarland), and the research by ZeMA was financed by HYDAC Filter Systems GmbH (Sulzbach, Germany).

The MoSeS-Pro project is sponsored by the German Federal Ministry of Education and Research in the call Sensor-based electronic systems for applications for Industrie 4.0 - SElekt I 4.0, funding code $16 \mathrm{ES} 0419 \mathrm{~K}$, within the framework of the German Hightech Strategy.

Edited by: Klaus-Dieter Sommer

Reviewed by: three anonymous referees

\section{References}

Akmal Johar, M. and König, A.: Case Study of an Intelligent AMR Sensor System with Self-x Properties, in: Soft Computing in Industrial Applications, edited by: Gaspar-Cunha, A., Takahashi, R., Schäfer, G., and Costa, L., Springer, Berlin Heidelberg, 337346, https://doi.org/10.1007/978-3-642-20505-7_30, 2011.

AMA Association for Sensors and Measurement (AMA Verband für Sensorik und Messtechnik e.V., originally Arbeitsgemeinschaft Messwertaufnehmer), available at: http://ama-sensorik.de/ en, last access: 10 March 2018.

Arnold, H.: Kommentar Industrie 4.0: Ohne Sensorsysteme geht nichts, available at: http://www.elektroniknet.de/messen-testen/ sonstiges/artikel/110776/ (last access: 10 March 2018), 2014.

Bastuck, M., Schütze, A., and Sauerwald, T.: A new approach to self-monitoring of amperometric oxygen sensors, Sensors and Actuators B 214, 218-224, https://doi.org/10.1016/j.snb.2015.02.116, 2015. 
Baur, T., Schütze, A., and Sauerwald, T.: Optimierung des temperaturzyklischen Betriebs von Halbleitergassensoren, Tech. Mess., 82, 187-195, https://doi.org/10.1515/teme-2014-0007, 2015.

Bryzek, J.: Roadmap for the Trillion Sensor Universe, iNEMI Spring Member Meeting and Webinar, Berkeley, CA, 2 April, available at: http://www-bsac.eecs.berkeley.edu/scripts/ show_pdf_publication.php?pdfID=1365520205 (last access: 10 March 2018), 2013.

Cachay, J. and Abele, E.: Developing Competencies for Continuous Improvement Processes on the Shop Floor through Learning Factories - Conceptual Design and Empirical Validation, Procedia CIRP, 3, 638-643, https://doi.org/10.1016/j.procir.2012.07.109, 2012.

Digitale Transformation in der Industrie, available at: http:// bmwi.de/DE/Themen/Industrie/industrie-4-0.html, last access: 10 March 2018.

Doms, M. and Slatter, R.: Magnetoresistive sensors for angle, position, and electrical current measurement in demanding environments, Proc. SPIE 2014, 9113, https://doi.org/10.1117/12.2049886, 2014.

Duda, R. O., Hart, P. E., and Stork, D. G.: Pattern classification, 2 Edn. Wiley, New-York, 2000.

Gassmann, O. and Kottmann, J.: Technologiemanagement in der Sensorik, Wissensmanagement, 8, 19-24, 2002.

GUM: Evaluation of measurement data - Guide to the expression of uncertainty in measurement, JCGM 100, available at: http:// www.bipm.org/en/publications/guides/gum.html (last access: 10 March 2018), 2008.

Helwig, N. and Schütze, A.: Intelligentes Condition Monitoring mit automatisierter Merkmalsgenerierung und -bewertung, in: XXVIII. Messtechnisches Symposium des Arbeitskreises der Hochschullehrer für Messtechnik, edited by: Schütze, A. and Schmitt, B., Shaker Verlag, Aachen, 121-128, https://doi.org/10.5162/AHMT2014/P1, 2014.

Helwig, N. and Schütze, A.: Detecting and compensating sensor faults in a hydraulic condition monitoring system. Proc. SENSOR 2015 - 17th International Conference on Sensors and Measurement Technology, Nuremberg, 19-21 May, available at: https://doi.org/10.5162/sensor2015/D8.1, 2015.

Helwig, N., Pignanelli, E., and Schütze, A.: Condition Monitoring of a Complex Hydraulic System Using Multivariate Statistics, Proc. I2MTC-2015 - 2015 IEEE International Instrumentation and Measurement Technology Conference, paper PPS1-39, Pisa, Italy, 11-14 May, available at: https://doi.org/10.1109/I2MTC.2015.7151267, 2015a.

Helwig, N., Klein, S., and Schütze, A.: Identification and quantification of hydraulic system faults based on multivariate statistics using spectral vibration features, Proc. Eng., 120, 1225-1228, https://doi.org/10.1016/j.proeng.2015.08.835, 2015 b.

Helwig, N., Schneider, T., and Schütze, A.: MoSeS-Pro: Modular sensor systems for real time process control and smart condition monitoring using XMR-technology, Proc. 14th xMRSymposium "Magnetoresistive Sensors and Magnetic Systems", Wetzlar, Germany, 21-22 March, 2017a.

Helwig, N., Merten, P., Schneider, T., and Schütze, A.: Integrated Sensor System for Condition Monitoring of Electromechanical Cylinders, MDPI Proceedings 2017, 1, 626, https://doi.org/10.3390/proceedings1040626, $2017 \mathrm{~b}$.
Hightech-Strategie: Industrie 4.0; available at: http://www. hightech-strategie.de/de/Industrie-4-0-59.php, last access: 10 March 2018.

iCM Hydraulics - Data-based intelligent condition monitoring for hydraulic systems; project funded in the EFI program of Saarland, subcontract by HYDAC Filter Systems GmbH, performed at Centre for Mechatronics and Automation gGmbH (ZeMA), 2013-2015.

Imkamp, D., Berthold, J., Heizmann, M., Kniel, K., Manske, E., Peterek, M., Schmitt, R., Seidler, J., and Sommer, K.-D.: Challenges and trends in manufacturing measurement technology the "Industrie 4.0" concept, J. Sens. Sens. Syst., 5, 325-335, https://doi.org/10.5194/jsss-5-325-2016, 2016.

Industrial Internet Consortium, available at: http://www. iiconsortium.org, last access: 10 March 2018.

Kitching, J., Donley, E. A., Knappe, S., Hummon, M., Dellis, A. T., Sherman, J., Srinivasan, K., Aksyuk, V. A., Li, Q., Westly, D., Roxworthy, B., and Lal, A.: NIST on a Chip: Realizing SI units with microfabricated alkali vapour cells, Journal of Physics: Conference Series, 723, 012056, https://doi.org/10.1088/17426596/723/1/012056, 2016.

MoSeS-Pro: Modulare Sensorsysteme für EchtzeitProzesssteuerung und smarte Zustandsbewertung für die Industrie 4.0, BMBF project funded in the funding area "Sensorbasierte Elektroniksysteme für Anwendungen für Industrie 4.0 (SElekt I4.0)", available at: http://www.moses-pro.de/ (last access: 10 March 2018), 2015-2018.

Marien, J. and Schütze, A.: Magnetic Microsensors: Quo vadis?, Proc. SENSOR 2009, II, 17-22, Nuremberg, 26-28 May, available at: https://doi.org/10.5162/sensor09/v2/a6.1, 2009.

Munter, P. J. A.: A low-offset spinning-current hall plate, Sensors and Actuators B, 22, 743-746, https://doi.org/10.1016/09244247(89)80069-X, 1990.

NIST-on-a-Chip Portal, available at: http://www.nist.gov/pml/ productsservices/nist-chip-portal, last access: 10 March 2018.

Ochs, T.: Selbstüberwachung und online Verifizierung von Sensordaten im Kraftfahrzeug, Übersichtsvortrag, 11. Dresdner Sensorsymposium 2013, Dresden, 9-11 December 2013.

Plattform Industrie 4.0, available at: http://www.plattform-i40. de/I40/Navigation/EN/Home/home.html, last access: 10 March 2018.

Reimann, P. and Schütze, A.: Sensor Arrays, Virtual Multisensors, Data Fusion, and Gas Sensor Data Evaluation, in: Gas Sensing Fundamentals, edited by: Kohl, C.-D. and Wagner, T., Springer Series on Chemical Sensors and Biosensors, Volume 15, 2014.

Schneider, T., Helwig, N., and Schütze, A.: Automatic feature extraction and selection for classification of cyclical time series data, Tech. Mess., 84, 198-206, https://doi.org/10.1515/teme2016-0072, 2017

Schüler, M., Sauerwald, T., and Schütze, A.: A novel approach for detecting HMDSO poisoning of metal oxide gas sensors and improving their stability by temperature cycled operation, J. Sens. Sens. Syst., 4, 305-311, https://doi.org/10.5194/jsss-4-305-2015, 2015.

Schütze, A.: Sensorik und Messtechnik im Industrie 4.0-Zeitalter, Plenarvortrag, 7. VDI-Fachtagung Messunsicherheit 2015 Messunsicherheit praxisgerecht bestimmen, Braunschweig, Germany, 19-20 November, 2015. 
Schütze, A. and Helwig, N.: Sensorik und Messtechnik für die Industrie 4.0 - (Sensors, instrumentation and measurement science for "Industrie 4.0"), Tech. Mess., 83, 208-218, https://doi.org/10.1515/teme-2016-0047, 2016.

Schütze, A. and Helwig, N.: Sensors 4.0 - Smart sensors and measurement technology enable Industry 4.0, Proc. 14th xMRSymposium "Magnetoresistive Sensors and Magnetic Systems", Wetzlar, Germany, 21-22 March, 2-8, 2017.

Semantic Sensor Network Ontology, available at: http://www.w3. org/2005/Incubator/ssn/ssnx/ssn, last access: 10 March 2018.

Sensor-Trends 2014 - Trends in zukunftsorientierten Sensortechnologien, edited by: AMA Fachverband für Sensorik, available at: http://www.ama-sensorik.de/fileadmin/Pubikationen/AMA Trendbericht_Langfassung[1].pdf (last access: 10 March 2018), 2010.

Sommer, K.-D. and Schütze, A.: Smart sensors \& networked digital measurement systems - Trends and challenges in industrial measurement and metrology, Keynote lecture, 46th Ann. Meas. Science Conf. 2016, Anaheim, USA, 23-25 March, 2016.
Stahl-Offergeld, M.: Robuste dreidimensionale Hall-Sensoren für mehrachsige Positionsmesssysteme, "Aktuelle Berichte aus der Mikrosystemtechnik - Recent Developments in MEMS”, Band 20, Shaker-Verlag, Aachen, 2011.

Stahl-Offergeld, M., Cichon, D., Hohe, H., and Schütze, A.: Offset Tracing in Hall Sensors by Integrated Temperature Coefficient Determination, Proc. SENSOR 2009, II, 59-64, https://doi.org/10.5162/sensor09/v2/a7.4, 2009.

Stahl-Offergeld, M., Ernst, R., Hohe, H.-P., and Schütze, A.: Process-independent Integrated Sensitivity Calibration of 3D Hall Sensors, EMSA 2010, the 8th European Conference on Magnetic Sensors and Actuators, Bodrum, Turkey, 4-7 July, 2010.

Walter, K.-D.: Wo bleibt der Sensor für Industrie 4.0?, available at: http://www.elektrotechnik.vogel.de/ wo-bleibt-der-sensor-fuer-industrie-40-a-529141/, last access: 10 March 2018. 